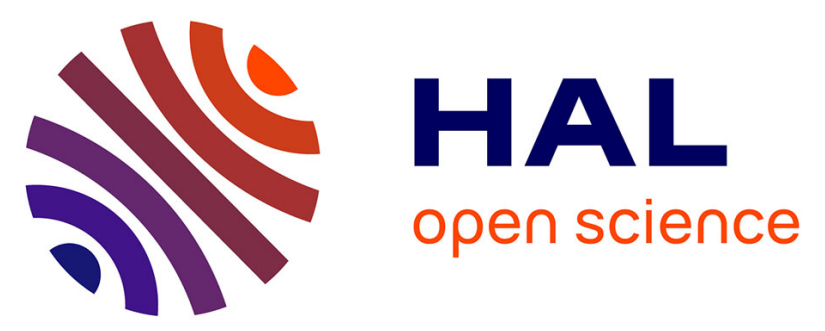

\title{
Propagation of Myocardial Fibre Architecture Uncertainty on Electromechanical Model Parameter Estimation: A Case Study
}

Roch Molléro, Dominik Neumann, Marc-Michel Rohé, Manasi Datar, Herve Lombaert, Nicholas Ayache, Dorin Comaniciu, Olivier Ecabert, Marcello Chinali, Gabriele Rinelli, et al.

\section{To cite this version:}

Roch Molléro, Dominik Neumann, Marc-Michel Rohé, Manasi Datar, Herve Lombaert, et al.. Propagation of Myocardial Fibre Architecture Uncertainty on Electromechanical Model Parameter Estimation: A Case Study. Functional Imaging and Modeling of the Heart, LNCS., Jun 2015, Maastricht, Netherlands. pp. 448-456, 10.1007/978-3-319-20309-6_51 . hal-01241896

\section{HAL Id: hal-01241896 \\ https://inria.hal.science/hal-01241896}

Submitted on 11 Dec 2015

HAL is a multi-disciplinary open access archive for the deposit and dissemination of scientific research documents, whether they are published or not. The documents may come from teaching and research institutions in France or abroad, or from public or private research centers.
L'archive ouverte pluridisciplinaire HAL, est destinée au dépôt et à la diffusion de documents scientifiques de niveau recherche, publiés ou non, émanant des établissements d'enseignement et de recherche français ou étrangers, des laboratoires publics ou privés. 


\title{
Propagation of Myocardial Fibre Architecture Uncertainty on Electromechanical Model Parameter Estimation: a Case Study
}

\author{
Roch Molléro ${ }^{1}$, Dominik Neumann ${ }^{3,4}$, Marc-Michel Rohé ${ }^{1}$, Manasi Datar ${ }^{3}$, \\ Hervé Lombaert ${ }^{1}$, Nicholas Ayache ${ }^{1}$, Dorin Comaniciu ${ }^{2}$, \\ Olivier Ecabert ${ }^{3}$, Marcello Chinali ${ }^{5}$, Gabriele Rinelli ${ }^{5}$, \\ Xavier Pennec ${ }^{1}$, Maxime Sermesant ${ }^{1, \star}$, and Tommaso Mansi ${ }^{2}$ \\ 1 Inria, Asclepios Research Project, Sophia Antipolis, France \\ 2 Siemens Corporate Technology, Imaging and Computer Vision, Princeton, NJ \\ 3 Siemens Corporate Technology, Imaging and Computer Vision, Erlangen, Germany \\ 4 Friedrich-Alexander-Universität, Pattern Recognition Lab, Erlangen-Nürnberg, \\ Germany \\ ${ }^{5}$ Ospedale Pediatrico Bambino Gesù, Rome, Italy
}

\begin{abstract}
Computer models of the heart are of increasing interest for clinical applications due to their discriminative and predictive power. However the personalisation step to go from a generic model to a patientspecific one is still a scientific challenge. In particular it is still difficult to quantify the uncertainty on the estimated parameters and predicted values. In this manuscript we present a new pipeline to evaluate the impact of fibre uncertainty on the personalisation of an electromechanical model of the heart from ECG and medical images. We detail how we estimated the variability of the fibre architecture among a given population and how the uncertainty generated by this variability impacts the following personalisation. We first show the variability of the personalised simulations, with respect to the principal variations of the fibres. Then discussed how the variations in this (small) healthy population of fibres impact the parameters of the personalised simulations.
\end{abstract}

\section{Introduction}

Cardiac modelling aims at understanding cardiac diseases (such as heart failure, dissynchrony or tachycardia), helping diagnosis and predicting cardiac response to therapy (e.g. cardiac resynchronization therapy, or radiofrequency ablation). In order to impact clinical practice, generic models have to be adjusted to a given patient, which is personalisation [1-4]. This is still a challenging part, and often computationally demanding, therefore most of the approaches are deterministic. However there are several sources of uncertainty, both due to the data and the models $[5,6]$. In this work we present the propagation of the uncertainty coming from the lack of knowledge on cardiac fibres for a given patient. Indeed, it is still

\footnotetext{
* Corresponding author: maxime.sermesant@inria.fr
} 


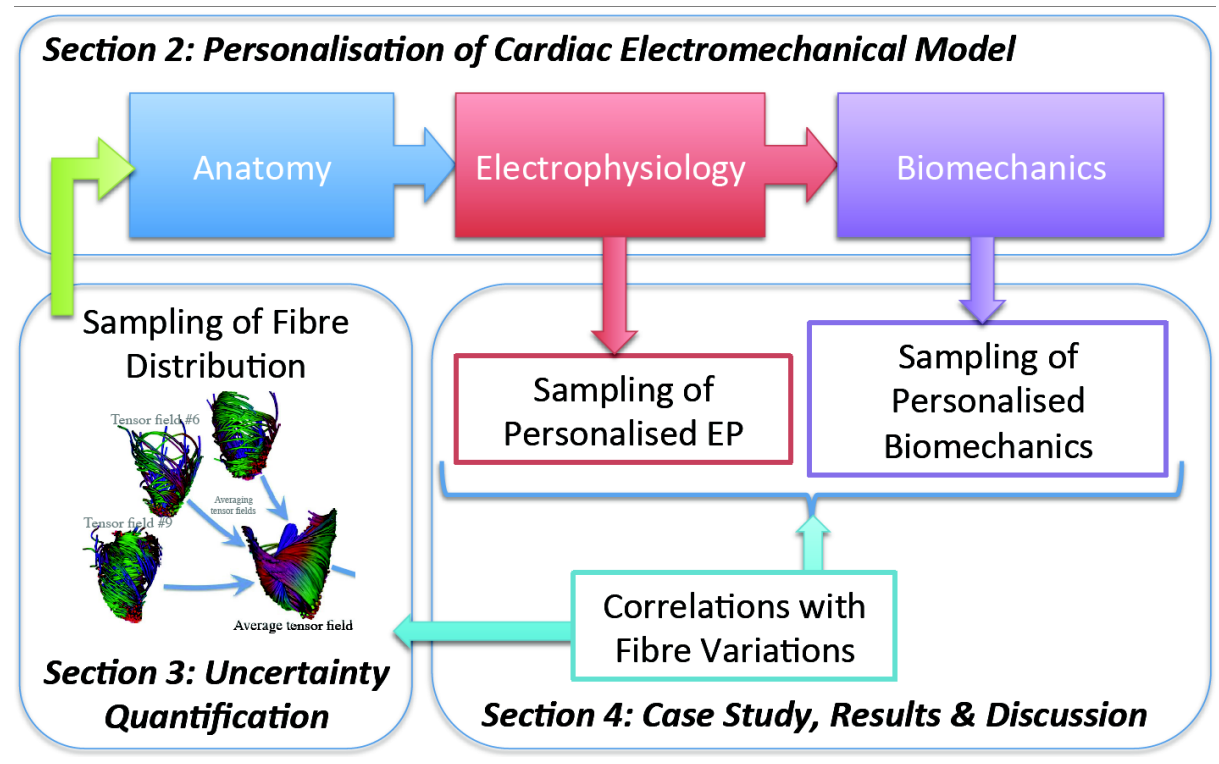

Fig. 1. Global scheme of fibre variability propagation along personalisation pipeline.

difficult to obtain measurements on the fibre architecture for a given patient in-vivo, therefore we have to rely on prior knowledge. In order to propagate this uncertainty, it has first to be quantified. This was done by computing statistics on a small population of healthy hearts (details in Sec. 3). Then the personalisation pipeline has to be efficient enough so that a sampling of this uncertainty can be propagated. Finally we obtained a sampling of the distribution of the parameters and personalised simulations (see Fig. 1).

We illustrated this method on a paediatric dilated cardiomyopathy case (details in 4.1). From a clinical standpoint, it is very difficult to predict the dramatic evolution of such rapidly-evolving case, even with advanced imaging. The aim of the project is to test if parameters derived from biophysical models could help predicting the outcome of such cases.

\section{Personalisation of Cardiac Electromechanical Model}

\subsection{Robust Segmentation of Myocardium from MRI}

Patient-specific heart morphology is obtained from short-axis cine magnetic resonance images (MRI). To that end, a robust, data-driven machine learning approach is employed [7] to estimate surface meshes of the left endocardium, left outflow tract, left epicardium, right endocardium, right outflow tract and right inflow tract. Each surface is estimated using marginal space learning and probabilistic boosting trees, constrained by a shape model learned from a database of 
hundreds of cases, thus ensuring inter-patient point correspondence. Next, each surface is tracked over the entire cine sequence using a combination of tracking by detection and tracking by registration. Finally, the surface meshes at middiastole are selected to generate a closed surface of the biventricular myocardium, which is transformed into a tetrahedral volume mesh for simulation ${ }^{6}$.

\subsection{Personalised Cardiac Electrophysiology Model}

Cardiac electrophysiology (EP) is modelled using the approach presented in [4]. Cardiac transmembrane potentials are calculated according to the mono-domain Mitchell-Schaeffer (MS) model as it offers a good compromise between model observability and fidelity (The equations are recalled in the supplementary material). In this study, we are mostly interested in two parameters: the time during which the ion channels are closed $\tau_{\text {close }}$, which captures action potential duration and is directly linked to the QT duration; and tissue diffusivity $c$, which determines the speed of the electrical wave propagation and is directly linked to the QRS duration. We model fast regional diffusivity for the left $c_{\mathrm{LV}}$ and right $c_{\mathrm{RV}}$ endocardium to mimic the fast conducting Purkinje network, and slower diffusivity $c_{\text {myo }} \leq c_{\mathrm{LV}}, c_{\text {myo }} \leq c_{\mathrm{RV}}$, for the myocardium. Transmembrane potentials are calculated using LBM-EP, a Lattice-Boltzmann method, which is coupled to a boundary element method approach to calculate the 12-lead cardiac electrocardiogram (ECG) resulting from the cardiac potentials [4]. The model is finally personalised like in $[8,9]$. BOBYQA, a constrained gradient-free optimization method is used to estimate tissue diffusivity and $\tau_{\text {close }}$ such that computed QRS duration, QRS electrical axis (EA) and QT duration match the measurements.

\subsection{Personalised Cardiac Mechanical Model}

The cardiac mechanical model is based on the Bestel-Clement-Sorine (BCS) model [10]. This model describes the heart as a Mooney Rivlin material, and model the stress along the cardiac fibres according to microscopic scale phenomena. Particularly, this model is compatible with the laws of thermodynamics and is able to model the Starling Effect. In this pipeline, it integrates a circulation model representing the 4 phases of the cardiac cycle (aortic pressure modelled by a 4-parameter Windkessel model), and takes the depolarization times and the action potential durations in each point of the mesh as an input to compute the mechanical contraction and relaxation of the myocardium (See a video illustration and a summary of the equations in the supplementary material).

As in [3], we only personalize the most influential and independent parameters which are the maximal contraction $\sigma$, the viscosity coefficient $\mu$, the Bulk Modulus $K$ and the Aortic peripheral resistance $R p$. The calibration is performed following [3]: after performing 9 simulations using some specific parameter values that lie in a distribution of acceptable physiological values, the Unscented Transform Algorithm finds in one iteration the set of parameters that best fit

\footnotetext{
${ }^{6}$ http://www.cgal.org - computational geometry algorithms library
} 
the observations within this distribution. In our case, the observations are the minimal LV volume and the time between the two moments the LV is at $50 \%$ of its contraction volume, both calculated from the cine MRI.

\section{Population-Based Uncertainty Quantification of Fibres}

\subsection{Variability Estimation in Atlas Space}

One often characterize the variability of a random vector by its mean and covariance matrix since these two first moments completely characterize the Gaussian distribution. However, in more than a few dimensions, the covariance matrix is too large to be computed robustly from only a few data observations. An alternative is to draw just a few samples from the population distribution, either by choosing randomly a number of points from the data observations, or more rationally by selecting a few points that describe the main subspace of variation in the data, for instance through Principal Component Analysis (PCA). Within this subspace one could describe the variability using a minimal number of points thanks to the so-called sigma-points at the vertices of a minimal simplex, originally designed for the Unscented-Kalman Filter [11]. However, it is often empirical observed that using symmetric points on all axes is significantly more accurate for underlying symmetric distributions. This is the approach we took in this study to quantify the variability of the fibre architecture.

We used $N=10$ ex-vivo DTI acquisitions of healthy human hearts, registered in the atlas space [12]. Both left and right ventricles images were generated with this atlas but due to the lower resolution of the right ventricle we chose to use this atlas only for the left ventricle part. On the right ventricle, we instead use a single DTI heart acquisition with high resolution done by Johns Hopkins University (JHU) [13]. Therefore we have no variability estimation of the fibres for the right ventricle.

To compute the mean DTI over the population and quantify the variability, we work in the Log-Euclidean space [14] rather than the standard Euclidean space. The mean DTI is $\bar{D}=\exp \left(\frac{1}{N} \sum_{i=1}^{N} \log \left(D^{(i)}\right)\right)$ and the data matrix of centred observations is $X=\left[\operatorname{vect}\left(\log D^{(1)}-\log \bar{D}\right) \ldots \operatorname{vect}\left(\log D^{(N)}-\right.\right.$ $\log \bar{D})]$. The PCA is obtained by diagonalizing the large covariance matrix $\Sigma=X X^{\top} /(N-1)$ or more efficiently we chose to compute the singular-value decomposition (SVD) of the data matrix $X=U \Lambda V^{T}$, where the $N \times N$ diagonal matrix $\Lambda$ encloses the square root of the eigenvalues of $\Sigma$. We choose to only study the first 3 eigenmodes $U_{i=1,2,3}$ which explain $59 \%$ of the variation of the log-tensors seen in the population. Also we noted that the higher modes being increasingly affected by the noise of the DTI acquisition, they increasingly describe the variability due to the noise and not the intrisic variability. For each mode, we compute two symmetric images representing the range of variation along the mode at plus or minus one standard deviation as: $M_{i, \pm}(x)=\exp \left(\log (\bar{D}(x)) \pm \sigma_{i} U_{i}(x)\right)$ (See video in supplementary materials). 


\subsection{From Atlas to Patient Space}

In order to relate the atlas space to the geometry of our target patient, we register the mesh of our patient to the mask of both the atlas (for the LV) and the JHU heart (for the RV) with a three-steps framework. First, the mask of the patient is aligned with the mask using a rigid landmark based registration method. Correspondences between the atlas and the target heart are manually checked. Secondly, we perform a similarity registration with five coarse levels and one fine level, each of which are composed of 10 iterations. Finally, we perform a diffeomorphic registration using diffeomorphic demons algorithm with $15 \times 10 \times 5 \times 5$ iterations (from coarsest to finest levels), a Gaussian smoothing factor of 2 in the regularization phase, and an interpolation for the moving image done with B-splines [15]. We then get the full diffeomorphic transformation for each one of our two initial atlases to the target patient mask.

We apply the transformation found in the previous step to the mesh of the patient. For each of the vertices, if the correspondence lies within the RV we use the JHU DTI-image whereas we use the mean or the sampled images of the Lombaert atlas if it lies within the LV. We take the mean (in the Log-Euclidean space) of the tensors of the 5-nearest voxels. The tensor value is then reoriented using the Finite Strain method, and the fibre orientation is taken as its first eigenvector [16]. The results of the fibres personalisation are 7 sets of fibres shown in Figure 2.

\section{Propagation of Fibre Uncertainty on a Case Study}

\subsection{Clinical Background}

The patient is a 16 years old male who had no family history of cardiac disease. After being admitted at the hospital for chest pain, evidence of reduced ejection fraction and dilated left ventricle led to a first diagnosis of myocarditis. A detailed echocardiographic examination performed 3 month later showed evidence of markedly increased trabeculae of the left ventricular apical and lateral walls, possibly suggesting the presence of left ventricular non-compaction. The MRI study did not confirm this diagnosis but only the idiopathic dilated cardiomyomathy. After 9 month of follow-up in the clinic, the patient was put on the national heart transplant list due to worsening conditions. The patient is now doing well at follow-up after transplant, and the pathology and histology testing at the hospital confirmed the diagnosis of idiopathic dilated cardiomyopathy.

\subsection{Goodness of Fit and variability after Personalization}

For each of the 7 tested fibre architectures we personalised EP and EM parameters as described in Sec. 2.2. High goodness of fit between observations and simulations were achieved for all instances : for the ECG, the maximum obtained errors after personalization are $0.2 \mathrm{~ms}$ for QRS, $2.9 \mathrm{~ms}$ for QT and $0.3^{\circ}$ for EA, which is well below 1\% of the measured values for QRS (96 ms) and 
QT (413 ms), and below $1 \%$ of the maximum possible error $\left(180^{\circ}\right)$ for EA $\left(5^{\circ}\right)$, respectively. Similary in terms of mechanics, the error between simulated and measured minimal volume and the time at $50 \%$ contraction are below $3 \%$.

After this step, we can observe the spatial variability of EP depolarisation times and end-diastolic strain between modes in Fig. 2. Although the main features of the ECG are the same, variations in local depolarization times can be up to $10 \mathrm{~ms}$ from one set of fibre to the mean fibre set due to the difference in current propagation. Interestingly we can notice for some of the sets a correlation between the peaks and zones of the variations of fibre orientation, with those of the depolarisation times, which would be interesting to investigate deeper.

\subsection{Uncertainty on the Model Parameters and Discussion}

Table 1 shows the values of the parameters after calibration for the mean fibre model, and their relative variation for each fibre set $(\mathrm{Mx}$ and $\mathrm{Px}$ are the two fibre sets representing the mode $\mathrm{x}$ as described in Sec. 3 , for $\mathrm{x}=1,2$ or 3 ).

For the EP parameters, we first note that $c_{\mathrm{RV}}$ varies the most although the fibres are fixed on this ventricle. This might be explained by the large changes in direction of depolarisation on the LV due to changes in fibre orientation, which would require the conductivity of the RV to vary as well to match the same EA. Logically, $\tau_{\text {close }}$ varies very little, since it is directly linked to the QT duration, that is not much affected by fibre orientation. Finally, the observed variabilities should be compared to the intrinsic uncertainty due to the parameter estimation process (quantified as high as $45 \%$ for $c_{\mathrm{RV}}$ in some cases) [4].

About the mechanical parameters, we can easily explain the variations of the $\mathrm{Rp}$ and the $\sigma$. It's indeed well known that the fibre architecture has a strong influence on the stroke volume and when we fix all the parameters, we see that the ejection fraction is maximal for the mean fibre, with the largest variations along the mode 2. To achieve the same level of ejection fraction with a less efficient set of fibre, the peripheral resistance must be lowered and the maximum constraction increased, which is what we observe for all the modes, (and in a larger range for the mode 2). The variations of $\mathrm{K}$ and $\mu$ are more challenging to interpret directly. They impact directly the slopes of contraction and relaxation phases, thus ensuring the fitting of the time at $50 \%$ contraction.

\section{Conclusion}

In this manuscript we detailed how a quantified uncertainty on myocardial fibres could be propagated along an efficient model personalisation pipeline. We presented the need to comprehensively quantify the influence of the parameters on the final output, and reversely to quantify their uncertainty when personalising models in order to fit clinical data. Atlases with mean and principal modes of variations are a good way to hierarchically represent the main directions of variability on quantities with many parameters such as vector or scalar fields. We used that method for the uncertainty on local fibre orientation in each point 


\begin{tabular}{llll}
\hline Mean & Mode 1 & Mode 2 & Mode 3 \\
\hline & & & \\
\hline
\end{tabular}
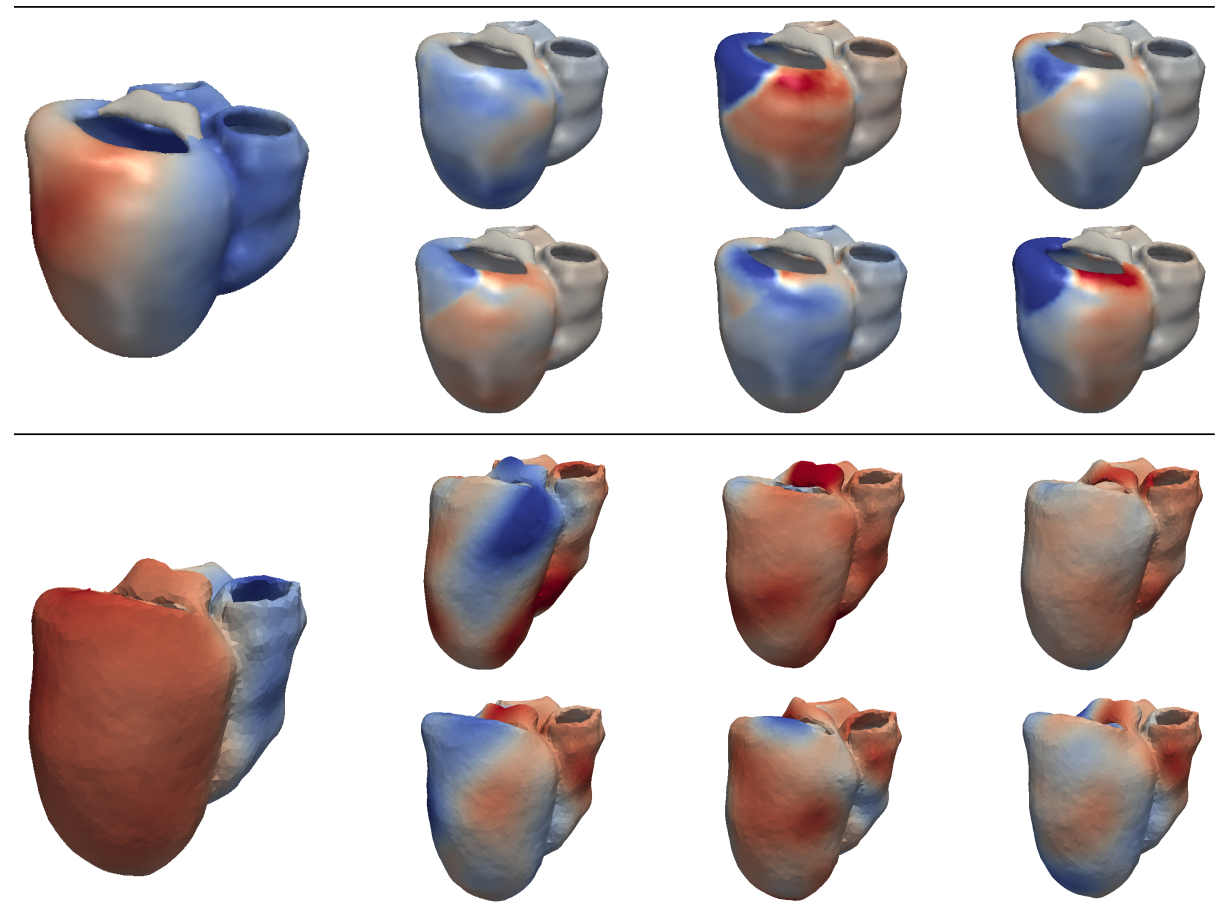

Fig. 2. Top left: Mean fibres. Top right: modes of variation plus (top) / minus (bottom) $\sigma$ coloured by angular variation w.r.t. the mean (from $0^{\circ}$ blue to $20^{\circ}$ red). Middle left: Mean depolarisation times after EP personalization; from blue (early) to red (late $\approx 100 \mathrm{~ms}$ ). Middle right: Variation from mean depolarisation times colouring from blue $(-10 \mathrm{~ms})$ to grey $(0 \mathrm{~ms})$ to red $(+10 \mathrm{~ms})$. Bottom left: Local strain at endsystole range from blue (high) to red (low). Bottom right: Variation for each different fibre modes after mechanical personalisation (blue: more contraction, red: less than on mean fibre).

of the heart, and assesed the variations of personalized parameters according to 
Table 1. Variability in estimated EP and EM model parameters after personalisation.

\begin{tabular}{l|cccccccc}
\hline $\begin{array}{l}\text { Parameter } \\
\text { Unit }\end{array}$ & $\begin{array}{c}c_{\text {myo }} \\
\mathrm{mm}^{2} / \mathrm{s}\end{array}$ & $\begin{array}{c}c_{\mathrm{LV}} \\
\mathrm{mm}^{2} / \mathrm{s}\end{array}$ & $\begin{array}{c}c_{\mathrm{RV}} \\
\mathrm{mm}^{2} / \mathrm{s}\end{array}$ & $\begin{array}{c}\tau_{\text {close }} \\
\mathrm{ms}\end{array}$ & $\begin{array}{c}\sigma \\
\mathrm{Pa}\end{array}$ & $\begin{array}{c}\mu \\
\mathrm{Pas}\end{array}$ & $\begin{array}{c}\mathrm{K} \\
\mathrm{Pa}\end{array}$ & $\begin{array}{c}\mathrm{Rp} \\
\mathrm{Pa} \mathrm{m}^{3} \mathrm{~s}\end{array}$ \\
\hline Mean & $1.21 \mathrm{e} 3$ & $4.70 \mathrm{e} 3$ & $1.83 \mathrm{e} 4$ & $2.09 \mathrm{e} 2$ & $4.74 \mathrm{e} 6$ & $2.29 \mathrm{e} 5$ & $2.01 \mathrm{e} 7$ & $1.9 \mathrm{e} 7$ \\
$\mathrm{P} 1$ & $-17.2 \%$ & $-14 \%$ & $-14.7 \%$ & $-0.48 \%$ & $+2.71 \%$ & $-5.84 \%$ & $-5.21 \%$ & $-26.7 \%$ \\
$\mathrm{M} 1$ & $+11.5 \%$ & $+10.6 \%$ & $+6.06 \%$ & $+0.14 \%$ & $+4.2 \%$ & $-8.54 \%$ & $-11.4 \%$ & $-30.9 \%$ \\
\hline P2 & $-0.82 \%$ & $+2.34 \%$ & $+24.2 \%$ & $-0.14 \%$ & $+2.4 \%$ & $-20.1 \%$ & $+4.86 \%$ & $-54.6 \%$ \\
$\mathrm{M} 2$ & $+3.28 \%$ & $+2.55 \%$ & $-30.9 \%$ & $-0.14 \%$ & $+0.86 \%$ & $-2.02 \%$ & $+10.6 \%$ & $-35.8 \%$ \\
\hline P3 & $+3.28 \%$ & $+7.02 \%$ & $-11.4 \%$ & $-0.14 \%$ & $+3.75 \%$ & $-2.87 \%$ & $-7.64 \%$ & $-34.4 \%$ \\
M3 & $-0.82 \%$ & $-7.66 \%$ & $+9.06 \%$ & $-0.57 \%$ & $+0.75 \%$ & $-12.6 \%$ & $-0.98 \%$ & $-20.7 \%$ \\
\hline
\end{tabular}

those uncertainty. Interestingly, if we have prior knowledge on some parameters of the heart, this method could reciprocally give us information on the fibre set with the higest probability.

Finally, several aspects of this pipeline could be further improved for a more general assessment of the uncertainty, in particular with a more advanced personalisation from clinical data (evolution of regional volumes, the whole flow curves..) and an extension of the us of atlases to regional parameters such as conductivity or stiffness maps.

Ackowledgements This work has been partially funded by the EU FP7-funded project MD-Paedigree (Grant Agreement 600932)

\section{References}

1. Xi, J., Lamata, P., Niederer, S., Land, S., Shi, W., Zhuang, X., Ourselin, S., Duckett, S.G., Shetty, A.K., Rinaldi, C.A., et al.: The estimation of patient-specific cardiac diastolic functions from clinical measurements. Medical image analysis $\mathbf{1 7}(2)(2013)$ 133-146

2. Krishnamurthy, A., Villongco, C.T., Chuang, J., Frank, L.R., Nigam, V., Belezzuoli, E., Stark, P., Krummen, D.E., Narayan, S., Omens, J.H., et al.: Patientspecific models of cardiac biomechanics. Journal of computational physics 244 (2013) 4-21

3. Marchesseau, S., Delingette, H., Sermesant, M., Ayache, N.: Fast parameter calibration of a cardiac electromechanical model from medical images based on the unscented transform. Biomechanics and modeling in mechanobiology, 12(4), 815$831(2013)$

4. Zettinig, O., Mansi, T., Neumann, D., Georgescu, B., Rapaka, S., Seegerer, P., Kayvanpour, E., Sedaghat-Hamedani, F., Amr, A., Haas, J., Steen, H., Katus, H., Meder, B., Navab, N., Kamen, A., Comaniciu, D.: Data-driven estimation of cardiac electrical diffusivity from 12-lead ECG signals. Medical Image Analysis 18(8) (2014) $1361-1376$

5. Neumann, D., Mansi, T., Georgescu, B., Kamen, A., Kayvanpour, E., Amr, A., Sedaghat-Hamedani, F., Haas, J., Katus, H., Meder, B., et al.: Robust image-based 
estimation of cardiac tissue parameters and their uncertainty from noisy data. In: Medical Image Computing and Computer-Assisted Intervention-MICCAI 2014. Springer (2014) 9-16

6. Konukoglu, E., Relan, J., Cilingir, U., Menze, B., Chinchapatnam, P., Jadidi, A., Cochet, H., Hocini, M., Delingette, H., Jaïs, P., Haïssaguerre, M., Ayache, N., Sermesant, M.: Efficient probabilistic model personalization integrating uncertainty on data and parameters: Application to eikonal-diffusion models in cardiac electrophysiology. Progress in Biophysics and Molecular Biology 107(1) (Oct 2011) 134-146

7. Wang, Y., Georgescu, B., Chen, T., Wu, W., Wang, P., Lu, X., Ionasec, R., Zheng, Y., Comaniciu, D.: Learning-based detection and tracking in medical imaging: A probabilistic approach. In: LNCVB. Volume 7. (2013) 209-235

8. Neumann, D., Mansi, T., Grbic, S., Voigt, I., Georgescu, B., Kayvanpour, E., Amr, A., Sedaghat-Hamedani, F., Haas, J., Katus, H., et al.: Automatic image-tomodel framework for patient-specific electromechanical modeling of the heart. In: Biomedical Imaging (ISBI), 2014 IEEE 11th International Symposium on, IEEE (2014) 935-938

9. Seegerer, P., Mansi, T., Jolly, M.P., Neumann, D., Georgescu, B., Kamen, A., Kayvanpour, E., Amr, A., Sedaghat-Hamedani, F., Haas, J., et al.: Estimation of regional electrical properties of the heart from 12-lead ECG and images. Volume 8896 of LNCS.

10. Chapelle, D., Le Tallec, P., Moireau, P., Sorine, M.: Energy-preserving muscle tissue model: formulation and compatible discretizations. International Journal for Multiscale Computational Engineering, 10(2) (2012)

11. Julier, S.J., Uhlmann, J.K.: A new extension of the kalman filter to nonlinear systems. In: Int. symp. aerospace/defense sensing, simul. and controls. Volume 3., Orlando, FL (1997) 3-2

12. Lombaert, H., Peyrat, J.M., Croisille, P., Rapacchi, S., Fanton, L., Clarysse, P., Delingette, H., Ayache, N.: Statistical analysis of the human cardiac fiber architecture from dt-mri. In Axel, L., Metaxas, D., eds.: Proceedings of FIMH Conference 2011. Volume 6666 of LNCS., Springer (May 2011) 171-179

13. Helm, P.A., Tseng, H.J., Younes, L., McVeigh, E.R., Winslow, R.L.: Ex vivo 3d diffusion tensor imaging and quantification of cardiac laminar structure. Magnetic Resonance in Medicine (2005)

14. Arsigny, V., Commowick, O., Ayache, N., Pennec, X.: A fast and log-Euclidean polyaffine framework for locally linear registration. Journal of Mathematical Imaging and Vision 33(2) (2009) 222-238

15. Vercauteren, T., Pennec, X., Perchant, A., Ayache, N.: Diffeomorphic demons: Efficient non-parametric image registration. NeuroImage 45(1, Supp.1) (March 2009) S61-S72

16. Peyrat, J.M., Sermesant, M., Pennec, X., Delingette, H., Xu, C., McVeigh, E.R., Ayache, N.: A computational framework for the statistical analysis of cardiac diffusion tensors: Application to a small database of canine hearts. IEEE Transactions on Medical Imaging 26(11) (November 2007) 1500-1514 